\title{
FOUR UNUSUAL CASES OF CONJUNCTIVITIS
}

BY

\author{
MAJOR. E. F. KING \\ R.A.M.C.
}

THESE four cases were of chronic conjunctivitis occurring in soldiers.

Though differing in detail they show certain features in common, which, I think, group them as a clinical entity, the pathogenesis of which, however, remains unsolved. I do not recall having seen similar cases in civilian practice or to have read a description of the condition in the literature.

The following are the clinical features common to each of the cases :

On preliminary examination slight ptosis was seen, together with a boggy fullness of the skin of both upper and lower lids. The eye tended to water and to be photophobic, but, in general, discharge was scanty, except during periods of secondary infection. The injection of the conjunctiva was largely confined to the posterior surface of the lids and the fornices, particularly the lower, and to the region of the plica and caruncle. This distribution of the hyperaemia was striking, and contrasted markedly with the relative whiteness of the ocular conjunctiva. The whole conjunctiva was somewhat oedematous and there was a diffuse milkiness, particularly in the lower fornix, reminiscent of spring catarrh, and presumably the result of epithelial hyperplasia.

In the upper fornix were a few scattered follicles. The two more severe cases developed subconjunctival fibrosis in the lower fornix, leading subsequently, in the case of one man, to marked contraction.

In two cases the cornea remained unaffected, in the other two a diffuse superficial keratitis was seen, extending over the greater part of the cornea; 'superficial vascularisation, occurred and the whole picture was very similar to a diffuse phlyctenular keratitis of children.

A striking feature is that each case remained entirely unilateral, there being no suggestion of involvement of the conjunctiva or cornea in the second eye. The condition was markedly persistent and chronic in its course. At the latter part of last year one case had persisted for eight, another for seven and two for four months. During these times the men had been completely incapacitated from their duties' and had spent the greater part of the time in hospital. There was seen during this period intermittent infection with secondary pyogenic organisms, accompanied by exacerbation of the keratitis and enlargement of the pre-auricular gland. 
The two severe cases, in which subconjunctival fibrosis occurred, were invalided out of the Army, the two others ultimately returned to duty, although the condition was still present and further relapses seemed probable.

The following are brief notes on each case :-

(a) Private H. (aged 27 years), Fig. 1.-Attended first giving the history of a burn to the left eye from a hot cinder. Examination showed some conjunctivitis and a small necrotic patch in the lower palpebral conjunctiva of the left eye. The condition

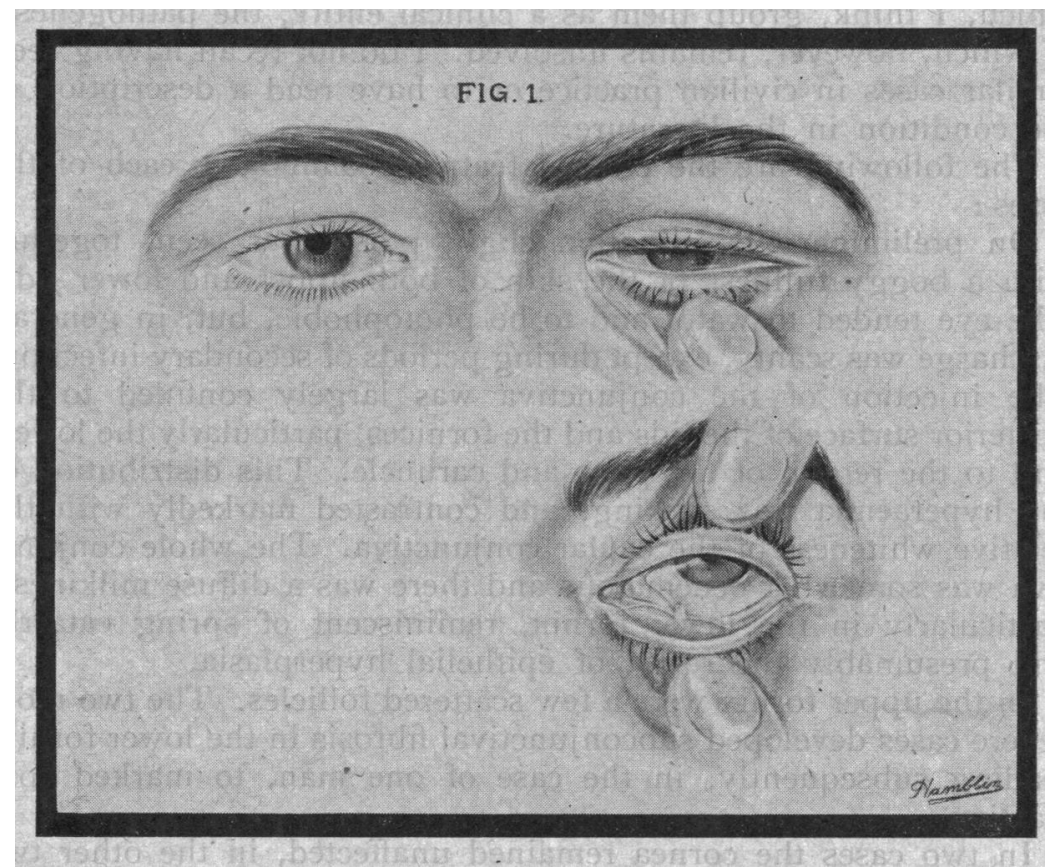

was slight and superficial and he was given out-patient treatment. Two weeks later he had developed oedema of the lids, particularly the lower, and conjunctival injection largely confined to the fornices. The ulcerated area in the lower fornix was still present and beneath it and the adjacent conjunctiva fibrosis was commencing. The pre-auricular gland on the same side was enlarged and tender. From this time he received continuous out and in-patient treatment until his discharge from the Army eight months later. During this period new foci of necrosis appeared in the lower fornix, and as each healed further subconjunctival scarring resulted. Finally, the condition appeared relatively quiescent, though there was still marked oedema of the lids and conjunctival injection. The diffuse fibrosis in the lower fornix appeared to be continuous with a 
generalised thickening of the lower tarsal plate. During the active period, secondary infection of the conjunctiva occurred from time to time, leading to muco-purulent discharge and superficial keratitis with small areas of corneal ulceration; these attacks subsided in the course of a few days.

General examination of the man revealed no abnormality. All blood investigations were normal. Conjunctival culture revealed staphylococcus aureus only. Inoculation of guinea-pigs with a piece of excised conjunctiva failed to reveal any evidence of tuberculosis.

Biopsy of the affected conjunctiva showed " marked increase of collagen fibres, extensive round-celled infiltration with plasma cells, lymphocytes and a few polymorphonuclear cells. Pronounced periarteritis and endarteritis of small vessels. A combination of hyperplasia and degenerative changes in the surface epithelium. No evidence of tuberculosis. The appearance being that of a sub-acute, non-specific inflammatory reaction.'

A large number of therapeutic agents were tried, none of which appeared to assist in any way. The attacks of secondary infection were helped by sulphonamides; which were used both locally and generally.

(b) PRIVAte F. (aged 29 years), Fig. 2.-The onset in this man was acute. There was considerable injection and chemosis, largely

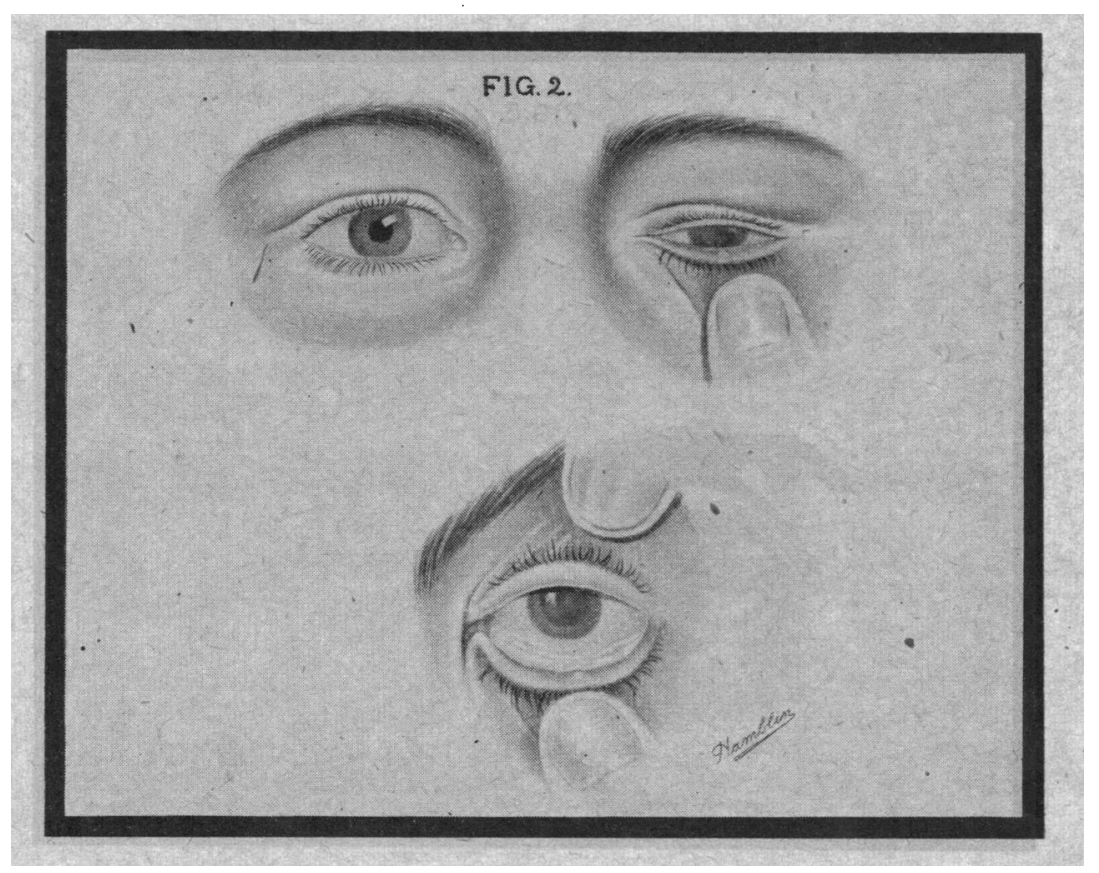


confined to the lower fornix. Both lids, but particularly the lower, were oedematous. There was enlargement of the pre-auricular gland on the affected side. When the acute phase had passed, the oedema of the lids and conjunctival injection persisted. The surface of the conjunctiva in the lower fornix developed a milky sheen, due to epithelial hyperplasia and beneath this some degree of subconjunctival fibrosis ensued, though there were no areas of conjunctival necrosis, such as were seen in the previous patient. A diffuse superficial keratitis slowly developed, leading at times to ulceration and ultimately to considerable vascularisation and scarring. After the acute onset the condition pursued a chronic indolent course, and after seven months he was invalided out of the Army:

As in the previous case, exhaustive tests, both local and genera! offered little help. Staphylococci only were grown from the conjunctival sac. Pathological examination of a piece of excised conjunctiva showed "a non-specific, sub-acute and chronic inflammatory process." All forms of treatment appeared to have little influence on the condition.

(c) Gunner H. (aged 29 years), Fig. 3.-The condition of this man was very similar to the last patient. The onset was less acute, though the oedema of the lids and conjunctival injection were marked, particularly in the lower fornix and on the plica. Hyperplasia on the conjunctival epithelium was seen, though there was

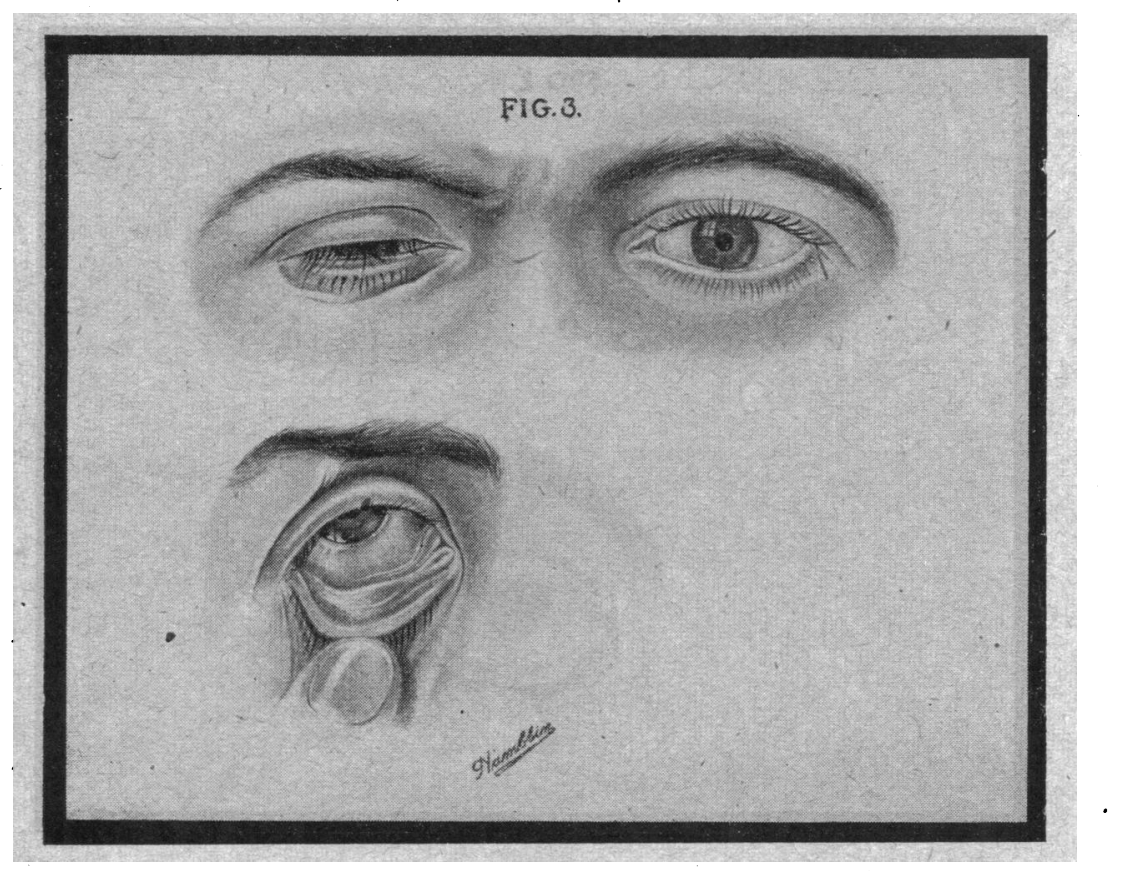


no subconjunctival fibrosis. The cornea remained unaffected throughout. After four months in hospital the condition had improved considerably, though recovery was not complete. $\mathrm{He}$ was able to return to duty.

As in the previous cases, investigations proved singularly unhelpful. Staphylococci only were grown from the conjunctiva. Biopsy of the,conjunctiva revealed sub-acute, non-specific inflammation. All the usual lines of treatment were tried with little apparent benefit.

(d) Signalman P. (aged 25 years), Fig. 4. - In this man the injection was at first so localised to the lower fornix that a provisional diagnosis of episcleritis was made. The oedema of the lower lid, conjunctival injection, and epithelial hyperplasia were very similar to the previous cases, though less severe. The cornea

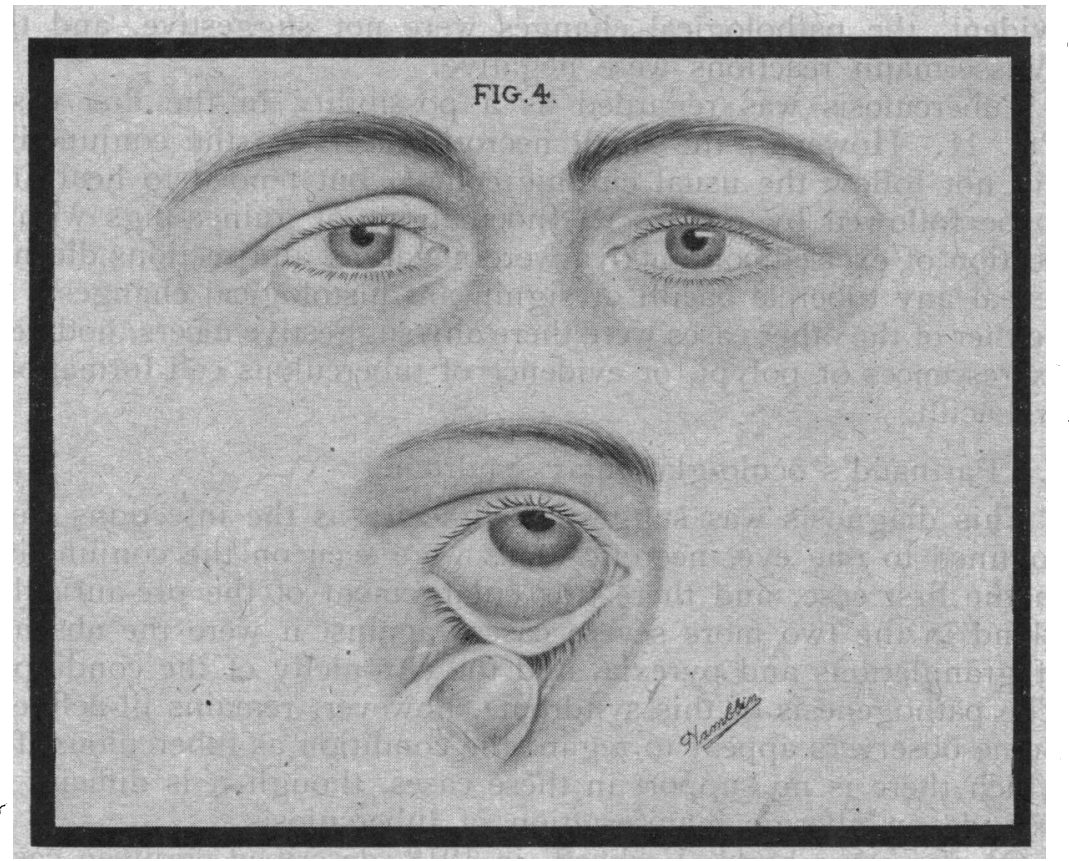

remained unaffected and subconjunctival fibrosis did not occur. After four months he was able to return to duty, though the condition had not entirely cleared up. Staphylococci were the only organisms in the conjunctival sac. Pathological examination of excised conjunctiva revealed, again, non-specific, sub-acute inflammation. All other investigations were negative. Treatment was along the usual lines. 


\section{Differential Diagnosis}

The following possibilities were considered :-

1. An allergic conjunctivitis.

A milky sheen on the conjunctiva, such as occurs in spring catarrh, was seen to a variable extent in all the cases, particularly in the lower fornix. Furthermore, the conjunctiva was in each case oedematous, shiny and dry; an appearance very similar to early atropine irritation. Neither of the men, however, gave a history of any general allergic sensitivity, nor was there any significant eosinophilia locally or in the blood.

2. A specific inflammation.

Syphilis was excluded with confidence. Neither patient gave a history of infection, no significant general clinical signs were evident, the pathological changes were not suggestive, and the Wassermann reactions were negative.

Tuberculosis was regarded as a possibility in the first case, Pte. H. However, the small necrotic ulcers on the conjunctiva did not follow the usual chronic course, but tended to heal and to be followed by new foci. Inoculations of guinea-pigs with a portion of excised conjunctiva were negative, and sections did not reveal any tubercle bacilli or significant histological changes. In neither of the other cases were there any suggestive ulcers, nodules, excrescences or polypi, or evidence of tuberculous cell formations or bacilli.

3. Parinaud's oculo-glandular syndrome.

This diagnosis was suggested clinically as the infections were confined to one eye, necrotic areas were seen on the conjunctiva in the first case, and there was enlargement of the pre-auricular gland in the two more severe cases; against it were the absence of granulations and pyrexia, and the chronicity of the condition. The pathogenesis of this syndrome, however, remains ill-defined. Some observers appear to regard the condition as tuberculous, for which there is no support in these cases, though it is difficult to exclude an allergic manifestation of tuberculosis.

On the other hand, Verhoeff, in 1918, described eighteen cases of Parinaud's conjunctivitis, which he regarded as a mycotic infection due to a filamentous organism previously undescribed. The essential pathological features of his cases were multiple necrotic focal areas beneath the epithelium, section of which showed masses of endothelial phagocytes, packed with chromatin granules and surrounded by chronic inflammatory cells. By special staining, the leptothrices were demonstrable in and around these focal lesions. The condition followed a relatively acute 
course and recovery occurred in a few weeks. Though the special staining technique for the organisms was not ádopted, I do not think that even the first of these cases (Pte. H.), in whom focal and necrotic lesions were seen in the conjunctiva, can be regarded as Verhoeff's leptothricosis conjunctivae for the course was essentially prolonged, marked subconjunctival scarring resulted, and sections of the conjunctiva failed to reveal any of the focal lesions he described.

4. Nutritional disturbances.

Some of the changes seen in the conjunctivae and corneae resembled those which have been described by Sydenstriker, Kruge and others as characteristic of vitamin $\mathrm{A}$ and riboflavin deficiences. They were, however, not typical and until the whole question of the ocular manifestations of nutritional disorders is clarified this suggestion must remain tentative.

\section{Conclusions}

This problem is one of monocular conjunctivitis, which persisted for many months. Biopsy of the conjunctiva showed marked changes, attributable to a non-specific, sub-acute inflammatory process.

In one case (Pte: H.), which was the most severe, small foci of necrosis in the lower fornix were seen, and subsequently marked contraction of the conjunctiva. In another (Pte. F.), there was no focal necrosis, but a mild subconjunctival fibrosis was apparent clinically: In the two other men (Gnr. H. and Sigmn. P.), the condition was less severe, no necrosis or scar contraction was apparent, and complete recovery was probable. It seems, howevèr, that the differences were of degree rather than kind, for, as suggested above, many points of clinical similarity were observed and the pathological report on the conjunctiva of each man was substantially the same.

The pathogenesis of the condition remains obscure and it would be of interest to learn if other surgeons have had the care of such cases.

\section{ANNOTATION}

\section{PURKYNĔ-A Pioneer in Ophthalmoscopy}

William Thau, of Boston, contributes a paper under the above heading to the Arch. of Ophthal., February, 1942. He comments in the first instance on the importance and amount of scientific knowledge hidden in the older literature, which is not 